\title{
Testing the early Late Ordovician cool-water hypothesis with oxygen isotopes from conodont apatite
}

\author{
PAGE C. QUINTON*†, STACEY LAW \\ ACHIM D. HERRMANN $\mid$, JOHN T. HAYNES $\$$ \& STEPHEN A. LESLIE \\ * Department of Geology, State University of New York, College at Potsdam, NY 13676, USA \\ \$Department of Geology and Environmental Science, James Madison University, 395 South High St., MSC 6903, \\ Harrisonburg, VA 22807, USA \\ §Department of Geological Sciences, University of Missouri, Columbia, MO 65211, USA \\ ๆDepartment of Geology and Geophysics, Coastal Studies Institute E301/302 Howe-Russell Geoscience Complex, \\ Louisiana State University, Baton Rouge, LA 70803, USA
}

(Received 12 February 2017; accepted 5 June 2017; first published online 2 August 2017)

\begin{abstract}
Latest Sandbian to early Katian sequences across Laurentia's epicontinental sea exhibit a transition from lithologies characterized as 'warm-water' carbonates to those characterized as 'coolwater'carbonates. This shift occurs across the regionally recognized M4/M5 sequence stratigraphic boundary and has been attributed to climatic cooling and glaciation, basin reorganization and upwelling of open ocean water, and/or increased water turbidity and terrigenous input associated with the Taconic tectophase. Documentation of oxygen isotopic trends across the M4/M5 and through bracketing strata provides a potential means of distinguishing among these alternative scenarios; however, oxygen isotopic records generated to date have failed to settle the debate. This lack of resolution is because $\delta^{18} \mathrm{O}$ records are open to multiple interpretations and potentially confounding factors related to local environmental conditions have not been tested by examining the critical interval in multiple areas and different depositional settings. To begin to address this shortcoming, we present new species-specific and mixed assemblage conodont $\delta^{18} \mathrm{O}$ values in samples spanning the M4/M5 boundary from the Upper Mississippi Valley, Alabama, and Virginia. The new results are combined with previous studies, providing a record of $\delta^{18} \mathrm{O}$ variability across SE Laurentia. The combined dataset allows us to test for regional trends at a resolution not previously available. Our results document $\mathrm{a} \sim 1.5 \%$ decrease in values across Laurentia instead of increasing $\delta^{18} \mathrm{O}$ values across the M4/M5 as predicted in various 'cool-water' scenarios. In short, these results do not support a shift to 'cool-water' conditions as an explanation for changes in early Katian carbonates across the M4/M5.
\end{abstract}

Keywords: Late Ordovician, oxygen isotopes, cool-water carbonates, conodonts, Katian.

\section{Introduction}

Latest Sandbian to early Katian sequences provide a record of major environmental changes across Laurentia's epicontinental sea that are commonly interpreted as representing the initiation of climatic cooling and glaciation $\sim 10$ Ma prior to the end Ordovician (Hirnantian) glacial interval (e.g. Lavoie, 1995; Pope \& Read, 1997; Pope \& Steffen, 2003; Saltzman \& Young, 2005). The proposed lithologic expression of the environmental changes is a shift from deposition of muddy, matrix-supported 'warm-water' carbonates to skeletally supported 'cool-water' carbonates across the early Late Ordovician M4/M5 sequence boundary (Holland \& Patzkowsky, 1996; Pope \& Read, 1997; Kolata, Huff \& Bergström, 2001). Additional observations that have been cited in favour of cooling across the M4/M5 include a migration of cool-water fauna into Laurentia, a $\sim 3 \%$ positive carbon isotope excursion (Guttenberg Isotope Carbon Excursion: GICE),

$\dagger$ Author for correspondence: quintopc@potsdam.edu and abundant phosphate and chert deposition above the M4/M5 sequence boundary (Holland \& Patzkowsky, 1996; Pope \& Read, 1997; Pope \& Steffen, 2003 [cf. Leslie \& Bergström, 2003]; Saltzman \& Young, 2005). Interpretations favouring early Katian climatic cooling pose some theoretical problems, however, and do not fit all of the available data. In particular, if temperature decrease is the dominant early Katian change, then the large magnitude of cooling at tropical to subtropical latitudes required to explain the lithological shift to interpreted 'cool-water' carbonates challenges existing thoughts about the relative stability of conditions in the tropics. Additionally, warm-water carbonates continued to accumulate in some regions within North America (e.g. parts of the mid-continent and the Upper Mississippi Valley), stratigraphically above the M4/M5 boundary (Bergström et al. 2010; Ettensohn, 2010), and the shift to deposition of carbonates with 'cool-water' features is diachronous even near the M4/M5 boundary (Lavoie \& Asselin, 1998; Dronov, 2013). Further, in contrast to a glacio-eustatic regression expected if the M4/M5 shift is associated with 
an initiation or expansion of continental glaciers (e.g. Bergström et al. 2010), the sequence boundary marks a relative rise in sea level across much of Laurentia (Holland \& Patzkowsky, 1996).

There are explanations for both the lithologic changes and the GICE that do not invoke early Katian cooling as a forcing mechanism. The Taconic tectophase of the Taconic Orogeny began during the Late Ordovician (Kolata, Huff \& Bergström, 2001; Ettensohn et al. 2002; Ettensohn, 2010). Nutrient availability, light penetration, terrigenous input, and/or salinity play important roles in determining carbonate production and, thus, influence lithology (Samankassou, 2002; Pedley \& Carannante, 2006). Some, if not all, of these variables likely changed in response to initial uplift of the Taconic highlands (including indirect responses related to modified circulation patterns within the basin). Increased upwelling of nutrient-rich open ocean water into the shallow epicontinental sea has also been invoked as a causal mechanism for changes in carbon cycling that resulted in the GICE (Young et al. 2005). In short, a tectonic model invoking uplift with associated crustal loading, increased weathering rates, and increased input of terrigenous material provides an alternative hypothesis to various cooling scenarios as an explanation for observed lithologic changes during the early Late Ordovician.

Geochemical-based palaeotemperature records can provide an independent perspective on environmental forcing. Several studies have used $\delta^{18} \mathrm{O}$ measurements from conodont apatite (a phase considered to be quite resistant to diagenetic overprinting due to the strong phosphate-oxygen bond, e.g. Luz, Kolodny \& Kovach, 1984; Wenzel, Lecuyer \& Joachimski, 2000; Joachimski et al. 2009) to estimate temperature trends in Laurentia's epicontinental sea during the early Katian (Buggisch et al. 2010; Herrmann, MacLeod \& Leslie, 2010; Rosenau, Herrmann \& Leslie, 2012). These studies have yielded somewhat contradictory interpretations. Two relatively high $\delta^{18} \mathrm{O}$ values measured near the base of the Katian in Oklahoma were cited as evidence of a brief cooling interval (Rosenau, Herrmann \& Leslie, 2012), whereas results from Minnesota, Iowa and Kentucky suggest warming during the early Katian followed by stable conditions through the remainder of the Stage (Buggisch et al. 2010). In addition, a low-resolution $\delta^{18} \mathrm{O}$ record spanning the entire Ordovician period suggests stable temperatures in the Katian (Trotter et al. 2008) while variation in $\delta^{18} \mathrm{O}$ values purported to be correlated with sea level change in samples from Kentucky and Anticosti Island were interpreted to support the presence of glacialinterglacial cycles throughout the Katian (Elrick et al. 2013). Attempting to integrate these $\delta^{18} \mathrm{O}$ records into a general palaeoclimatic scenario is complicated by the fact that these studies were conducted at different scales and generally present data from a few, closely spaced sections or composites of sections without significant time overlap. It is therefore difficult to rule out a role for local influences on observed $\delta^{18} \mathrm{O}$ trends that could attenuate or obscure the signature of global trends.

Change in the oxygen isotopic composition of the seawater $\left(\delta^{18} \mathrm{O}_{\mathrm{sw}}\right)$ from which the apatite precipitates is a major confounding factor in palaeotemperature estimates (e.g. Longinelli, 1965; Kolodny, Luz \& Navon, 1983; Luz, Kolodny \& Kovach, 1984). The $\delta^{18} \mathrm{O}_{\mathrm{sw}}$ value of a water mass can vary on local and regional scales due to differences in evaporation/precipitation rates and freshwater input or, globally, due to largescale climatic forcing including the volume of glacial ice. To constrain the relative contribution of temperature to an observed shift in $\delta^{18} \mathrm{O}$ values, it is necessary either to independently determine changes in $\delta^{18} \mathrm{O}_{\mathrm{sw}}$ (a difficult proposition) or to estimate local control on $\delta^{18} \mathrm{O}_{\mathrm{sw}}$ by documenting $\delta^{18} \mathrm{O}$ trends across multiple sections separated by distances that are long relative to likely gradients in $\delta^{18} \mathrm{O}_{\mathrm{sw}}$.

In this study we take the latter approach. We measured $\delta^{18} \mathrm{O}$ values from species-specific and mixed conodont assemblages from six sections across eastern North America to test for consistent $\delta^{18} \mathrm{O}_{\text {sw }}$ patterns across the region. The compiled $\delta^{18} \mathrm{O}$ record can be compared to predicted patterns of models that have been proposed. If the 'cool-water' interpretation of early Katian carbonates is correct, an increase in oxygen isotopic values is expected to correlate with the change in carbonate lithology across the M4/M5. Similarly, if the GICE records a net burial of organic carbon (and thus drawdown of atmospheric $\mathrm{CO}_{2}$ levels), an increase in oxygen isotopic values is expected to begin within or shortly above that carbon isotope excursion. Either of these isochronous increases in $\delta^{18} \mathrm{O}$ values would be consistent with global cooling and/or glaciation, whereas a diachronous increase could indicate an important role for cooling related to oceanographic changes such as an incursion and/or upwelling of cooler sub-thermocline water with many potential ultimate causes (e.g. basin deepening and/or changes in ocean circulation). Finally, and in marked contrast to the first three, stable or decreasing $\delta^{18} \mathrm{O}$ values would argue against cooling as a primary driver of observed lithological and geochemical changes during the early Katian.

\section{Materials and methods}

\section{2.a. Study sections}

During the Late Ordovician, Laurentia was rotated clockwise $\sim 45^{\circ}$ relative to modern geographic position and was in a subtropical latitude in the southern hemisphere (Torsvik \& Cocks, 2013). Much of E Laurentia was covered in a shallow epicontinental sea bounded by the Taconic highlands to the SE and the Transcontinental Arch to the NE. Connection to the Iapetus Ocean was established along the SE margin of the carbonate platform.

Data are presented from six sections that span the seaway along a roughly $\mathrm{N}-\mathrm{S}$ transect and represent 


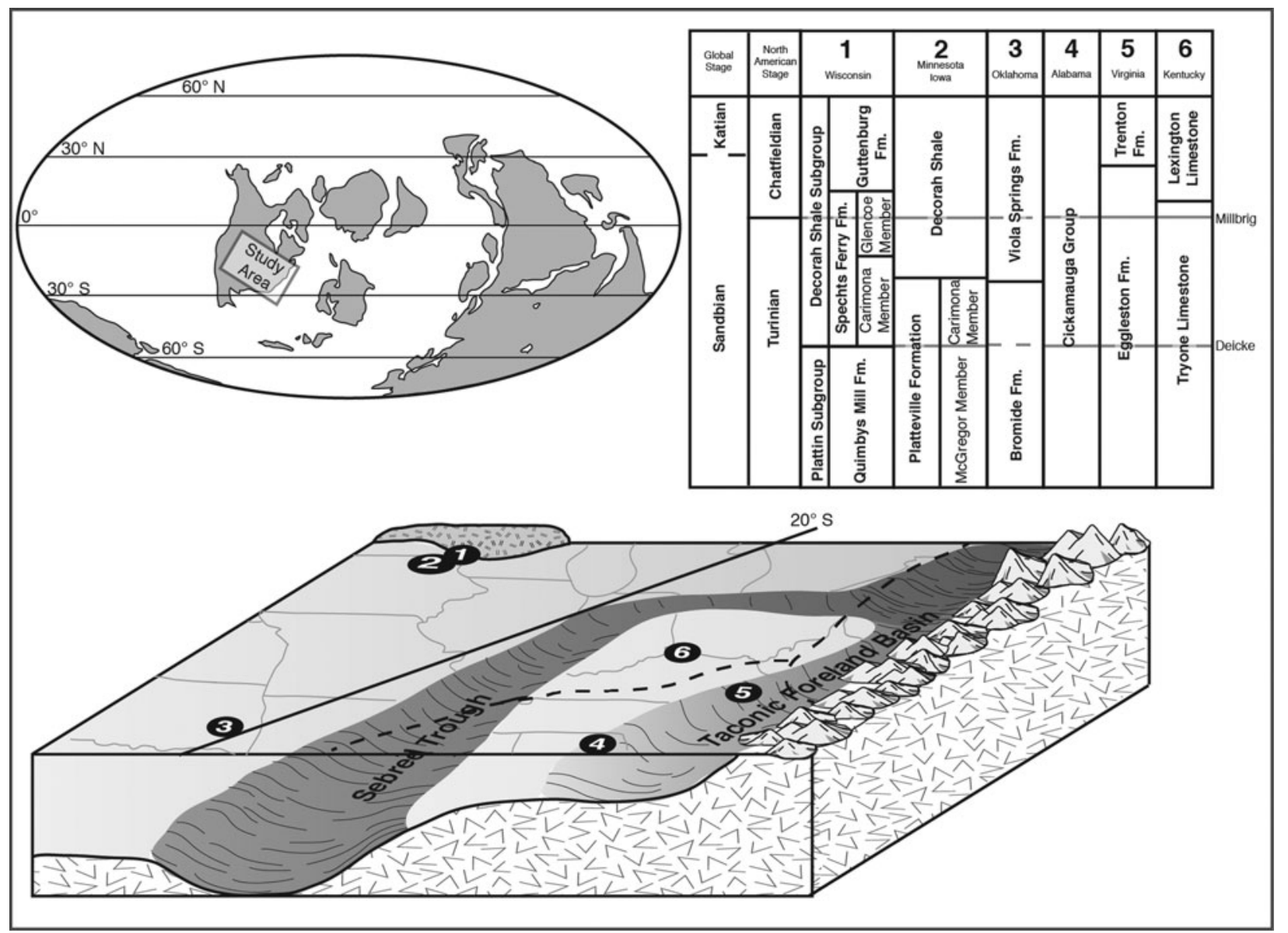

Figure 1. Global palaeogeographic reconstruction of the Late Ordovician (modified from Torsvik \& Cocks, 2013). The position of previously documented oxygen isotopic records spanning the M4/M5 (Buggisch et al. 2010; Rosenau, Herrmann \& Leslie, 2012) and sections from this study are identified on a generalized reconstruction of the bathymetry of the epicontinental sea (adapted from Brett et al. 2004). Water mass boundaries as identified by Holmden et al. (1998) are indicated with black dotted lines. Regional stratigraphy and position of the Deicke and Millbrig K-bentonite is provided for each composite section (adapted from Leslie, 2000).

different depositional settings within the shallow tropical epicontinental sea that covered much of North America (Fig. 1). The sections studied are the Tidwell Hollow and Red Mountain Expressway sections in Alabama $\left(33.87806^{\circ} \mathrm{N}, 86.57555^{\circ} \mathrm{W}\right.$ and $33.49598^{\circ} \mathrm{N}$ $\left.86.78822^{\circ} \mathrm{W}\right)$, the Dickeyville section in Wisconsin $\left(42.640586^{\circ} \mathrm{N} 90.57556^{\circ} \mathrm{W}\right)$, the Decorah section in Iowa $\left(43.29021^{\circ} \mathrm{N} 91.76561^{\circ} \mathrm{W}\right)$, the Rochester section in Minnesota $\left(43.97207^{\circ} \mathrm{N} 91.38358^{\circ} \mathrm{W}\right)$ and the Hagan section in Virginia $\left(36.28333^{\circ} \mathrm{N} 86.69250^{\circ} \mathrm{W}\right)$. Stratigraphic details for the Alabama sections can be found in Quinton et al. (2016a), for the Decorah section in Emerson et al. (2005), and the Rochester and Hagan sections in Leslie (1995). All sampled sections span the Sandbian-Katian boundary and contain the Deicke and Millbrig K-bentonites (Haynes, 1994; Kolata, Huff \& Bergström, 1996, 1998; Sell et al. 2015). The conodont fauna has previously been described for the Tidwell Hollow, Red Mountain Expressway, Decorah, Rochester and Hagan sections (Hall, Bergström \& Schmidt, 1986; Leslie, 1995, 2000; Quinton et al. 2016a ). In addition, these sections have been placed into the Midcontinent biostratigraphic framework (Leslie, 1995, 2000; Quinton et al. 2016a).
One complicating factor in attempting to understand the nature of environmental changes across M4/M5 is that the proposed stratigraphic position of the boundary among sections is inconsistent relative to other chronostratigraphic data (e.g. Sell et al. 2015). These chronostratigraphic markers include the Deicke and Millbrig K-bentonites, altered volcanic ash beds that have been recognized and correlated throughout eastern North America, and conodont biozones. For example, the base of the Plectodina tenuis zone has been identified above the Millbrig K-bentonite in some sections and below the Millbrig K-bentonite in other sections. The M4/M5 boundary has also been proposed to be above the Millbrig K-bentonite in some sections and below it in others. Further complication is added by the fact that the P. tenuis zone has been recognized both above and below the M4/M5 in different sections. These inconsistencies suggest at least three possibilities: (1) that the sequence boundary is diachronous (and thus not eustatic), (2) that the sequence boundary has been misidentified in some sections, or (3) that the sequence boundary is correctly located and isochronous but that other chronostratigraphic markers have been incorrectly located and/or identified in some 
sections (e.g. Kolata, Huff \& Bergström, 2001; Brett et al. 2004; Sell et al. 2015). Because ash deposited from a given eruption should provide an isochronous event bed where present (an assumption that is not necessarily true for biostratigraphic events or lithologic shifts), we use the Deicke and Millbrig K-bentonites to correlate among sections in addition to the stratigraphic position of the M4/M5. Unrecognized reworking or the presence of additional bentonites in a given section can be sources of uncertainty, but the correlations used are supported by phenocryst mineralogy in the study sections (e.g. Haynes, 1994; Sell et al. 2015).

\section{2.b. Oxygen isotope samples and methods}

Limestone samples of $\sim 5 \mathrm{~kg}$ and shale samples of $\sim 2 \mathrm{~kg}$ were collected at selected horizons in the Tidwell Hollow, Red Mountain Expressway, Decorah, Dickeyville and Rochester sections. To maintain consistency between sections, sampling was performed at regular stratigraphic intervals $(1-0.5 \mathrm{~m}$ intervals) all measured relative to the Millbrig or Deicke Kbentonite. To control for possible bias related to lithology, samples were also collected at significant changes in lithology.

Limestone samples were dissolved using either a $\sim 10 \%$ acetic solution or a $\sim 10 \%$ buffered formic solution following standard dissolution and extraction procedures (e.g. Jeppsson \& Anehus, 1995; Jeppsson et al. 1999; Quinton et al. 2016b). Shale samples were disaggregated by soaking in kerosene followed by a water and Calgon solution. All samples were washed on a 63 micron screen, and apatite microfossils were concentrated using heavy liquid (either tetrabromoethane or lithium heteropolytungstate: LST Heavy Liquid). Conodont elements and brachiopod shell fragments were isolated and identified using a binocular microscope. Conodonts used to document $\delta^{18} \mathrm{O}$ values for the Hagan section are from previous collections (Leslie, 1995, 2000).

Conodont elements are composed of highly crystalline bioapatite that is relatively resistant to alteration of the $\delta^{18} \mathrm{O}$ values in phosphate-bound oxygen (Kolodny \& Luz, 1991; Wenzel, Lecuyer \& Joachimski,. 2010; MacLeod, 2012). The conodont elements analysed in this study are well preserved. The elements lack evidence of overgrowths and have low conodont colour alteration index (CAI) values. CAI values range from 1 to 1.5, with the exception of the two sections from Alabama where elements have a CAI of 3. Together these observations suggest that measured $\delta^{18} \mathrm{O}$ values should be close to the primary oxygen isotopic ratios of the conodont elements, but diagenetic alteration cannot be completely ruled out.

To assess the extent and direction of any diagenetic shifts in the study sections, we measured phosphate $\delta^{18} \mathrm{O}$ values from co-occurring linguliform brachiopod shells. Bioapatite from linguliform brachiopod shells is generally thought to be more susceptible to alteration than conodont elements (Wenzel, Lecuyer \& Joachim- ski, 2000). This difference is due to the fact that brachiopods build their shells out of alternating layers of generally porous apatite and organic tissue and this architecture seems to have provided a relatively attractive substrate for microbial colonization. Measured oxygen isotopic values from brachiopod shells thus provide a geochemical taphonomic control on the direction and magnitude of local diagenetic overprints (Bassett et al. 2007; Hughes et al. 2015).

Previous conodont oxygen isotopic studies have documented significant variability in measured $\delta^{18} \mathrm{O}$ values, likely due to palaeoecological differences among conodont taxa, incomplete time averaging, and/or differences in diagenetic susceptibility among element types (Trotter et al. 2007; Rosenau, Herrmann \& Leslie, 2012; Sun et al. 2012; Quinton \& MacLeod, 2014; Quinton et al. 2016b). To reduce potential bias related to these factors, oxygen isotopic measurements were performed both on species-specific (Belodina compressa, Curtognathus sp., Drepandoistodus suberectus, Icriodella sp., Oulodus serratus, Panderodus gracilis, Phragmodus cognitus, Phragmodus undatus, Plectodina aculeta, Polyplacognathus ramosus) and mixed conodont assemblages. When possible, multiple oxygen isotopic measurements were generated for an individual sample horizon to increase confidence that values reflect the average for that stratigraphic horizon. Finally, oxygen isotopic values were also measured on brachiopod shell fragments to constrain digenetic influences. The $\mathrm{PO}_{4}{ }^{3-}$ anion was isolated using techniques modified from LaPorte et al. (2009) and described by Quinton \& MacLeod (2014). All samples were analysed on a continuous flow Thermo-Finnigan Delta Plus XL gas source mass spectrometer connected to a ThermoFinnigan TC-EA (high-temperature conversion - elemental analyzer) at $1400^{\circ} \mathrm{C}$ with an external precision of $\pm 0.3 \%$ ( 1 standard deviation) based on replicate analyses of NBS-120c. Values are reported in per mil notation (\%o) on the Vienna Standard Mean Ocean Water (VSMOW) scale and correspond to a NBS-120c value of $21.7 \%$. Oxygen isotopic values from previous studies (Buggisch et al. 2010; Roseneau et al. 2012) have been renormalized to a NBS-120c value of $21.7 \%$. Replicate analyses were performed for samples that yielded $>0.800 \mathrm{mg}$ of silver phosphate crystals (106 out of 257 samples). Reproducibility for these replicates was generally better than reported external precision (Table 1). For these samples (those with replicate analyses) the value reported is the average of the replicate $\delta^{18} \mathrm{O}$ measurements; for samples without replicates, the value measured is reported (Figs 2-4).

\section{2.c. Carbon isotope samples and methods}

To place our oxygen isotopic results within the general chemostratigraphic framework for the late Sandbian - early Katian, we measured co-occurring bulk carbonate and organic carbon $\delta^{13} \mathrm{C}$ values for the two 
Table 1. Conodont oxygen isotopic results for the Decorah section in Iowa $\left(43.29021^{\circ} \mathrm{N}, 91.76561^{\circ} \mathrm{W}\right)$, the Rochester section in Minnesota $\left(43.97207^{\circ} \mathrm{N}, 91.38358^{\circ} \mathrm{W}\right)$, the Dickeyville section in Wisconsin $\left(42.640586^{\circ} \mathrm{N}, 90.57556^{\circ} \mathrm{W}\right)$, the Hagen section in Virginia $\left(36.28333^{\circ} \mathrm{N}, 86.69250^{\circ} \mathrm{W}\right)$ and the Red Mountain Expressway and Tidwell Hollow sections in Alabama $\left(33.49598^{\circ} \mathrm{N}, 86.78822^{\circ} \mathrm{W}\right.$ and $\left.33.87806^{\circ} \mathrm{N}, 86.57555^{\circ} \mathrm{W}\right) .^{*}$. Averages are calculated from $\delta^{18} \mathrm{O}$ values of replicate analyses of silver phosphate crystals. One standard deviation $(\sigma)$ is calculated for samples with replicate analyses. Conodont species analysed include Belodina compressa, Curtognathus sp., Drepandoistodus suberectus, Icriodella superba, Oulodus serratus, Panderodus gracilis, Phragmodus cognitus, Phragmodus undatus,

Plectodina aculeta and Polyplacognathus ramosus

\begin{tabular}{|c|c|c|c|c|c|c|c|}
\hline Sample ID & metres* & Avg. & $\delta^{18} \mathrm{O}$ & $\delta^{18} \mathrm{O}$ & $\delta^{18} \mathrm{O}$ & $\sigma$ & Description \\
\hline \multicolumn{8}{|l|}{ Decorah } \\
\hline-10 & 15.49 & 18.9 & 18.9 & & & & Mixed conodont \\
\hline-9 & 13.94 & 19.0 & 19.0 & & & & D. suberectus \\
\hline-9 & 13.94 & 18.3 & 18.4 & 18.3 & & 0.1 & P. gracilis \\
\hline-9 & 13.94 & 18.9 & 18.9 & & & & P. undatus \\
\hline-8 & 12.34 & 18.2 & 18.4 & 17.9 & 18.5 & 0.3 & D. suberectus \\
\hline-8 & 12.34 & 18.3 & 18.6 & 17.9 & & 0.5 & P. undatus \\
\hline-7 & 8.91 & 18.1 & 17.7 & 18.3 & 18.3 & 0.3 & D. suberectus \\
\hline-7 & 8.91 & 18.8 & 18.8 & & & & P. undatus \\
\hline-6 & 6.55 & 18.5 & 18.6 & 18.3 & 18.5 & 0.2 & D. suberectus \\
\hline-6 & 6.55 & 18.5 & 18.5 & 18.5 & & 0.0 & P. ramosus \\
\hline-5 & 4.99 & 19.0 & 19.1 & 18.7 & 19.1 & 0.2 & D. suberectus \\
\hline-5 & 4.99 & 18.2 & 18.2 & & & & P. gracilis \\
\hline-5 & 4.99 & 19.2 & 19.1 & 19.2 & & 0.1 & P. undatus \\
\hline-4 & 4.64 & 19.1 & 19.1 & & & & D. suberectus \\
\hline-4 & 4.64 & 19.3 & 19.3 & & & & P. undatus \\
\hline-3 & 4.29 & 19.5 & 19.4 & 19.5 & & 0.1 & D. suberectus \\
\hline-3 & 4.29 & 18.9 & 18.9 & & & & P. gracilis \\
\hline-3 & 4.29 & 18.9 & 18.9 & & & & P. undatus \\
\hline-2 & 3.99 & 19.3 & 19.3 & & & & P. undatus \\
\hline-2 & 3.99 & 19.1 & 19.1 & & & & Mixed conodont \\
\hline-1 & 3.89 & 18.9 & 18.9 & & & & Mixed conodont \\
\hline-1 & 3.89 & 19.4 & 19.4 & & & & Brachiopod \\
\hline 1 & 3.75 & 18.9 & 18.9 & & & & Mixed conodont \\
\hline 2 & 3.70 & 18.9 & 18.9 & & & & P. undatus \\
\hline 2 & 3.70 & 19.7 & 19.7 & & & & Mixed conodont \\
\hline 3 & 3.65 & 19.0 & 19.0 & & & & Mixed conodont \\
\hline 4 & 3.61 & 18.8 & 18.8 & & & & P. undatus \\
\hline 4 & 3.61 & 18.9 & 18.9 & & & & Mixed conodont \\
\hline 5 & 3.54 & 19.4 & 19.4 & 19.4 & & 0.0 & P. undatus \\
\hline 5 & 3.54 & 19.1 & 19.0 & 19.2 & & 0.1 & Mixed conodont \\
\hline 6 & 3.33 & 18.8 & 18.8 & & & & P. undatus \\
\hline $3-1 L$ & 2.90 & 18.4 & 18.4 & & & & P. undatus \\
\hline $3-1 L$ & 2.90 & 19.0 & 19.0 & & & & Mixed conodont \\
\hline $3-0.5 \mathrm{M}$ & 2.82 & 19.1 & 19.1 & & & & Mixed conodont \\
\hline $3-2 \mathrm{~L}$ & 2.52 & 18.8 & 19.3 & 18.3 & & 0.7 & D. suberectus \\
\hline $3-2 \mathrm{~L}$ & 2.52 & 19.5 & 19.5 & & & & P. undatus \\
\hline $3-1.0 \mathrm{M}$ & 2.40 & 19.2 & 19.2 & & & & D. suberectus \\
\hline $3-1.0 \mathrm{M}$ & 2.40 & 18.8 & 18.8 & 18.7 & & 0.0 & O. serratus \\
\hline $3-1.0 \mathrm{M}$ & 2.40 & 19.5 & 19.8 & 19.1 & & 0.5 & Mixed conodont \\
\hline $3-2 \mathrm{LB}$ & 1.38 & 19.8 & 19.9 & 19.7 & & 0.1 & D. suberectus \\
\hline $3-2 \mathrm{LB}$ & 1.38 & 19.1 & 19.1 & 19.0 & & 0.1 & P. aculeata \\
\hline $3-2 \mathrm{LB}$ & 1.38 & 19.4 & 19.5 & 19.3 & & 0.2 & P. gracilis \\
\hline 3-2LB & 1.38 & 18.9 & 18.9 & 18.9 & & 0.0 & O. serratus \\
\hline $3-4 \mathrm{~L}$ & 0.69 & 19.3 & 19.4 & 19.3 & & 0.0 & D. suberectus \\
\hline $3-4 \mathrm{~L}$ & 0.69 & 19.6 & 19.6 & 19.5 & & 0.1 & P. cognitus \\
\hline $3-4 \mathrm{~L}$ & 0.69 & 18.6 & 18.6 & & & & $P$ gracilis \\
\hline $3-2.5 \mathrm{M}$ & 0.69 & 19.0 & 19.3 & 18.6 & 19.2 & 0.3 & D. suberectus \\
\hline $3-2.5 \mathrm{M}$ & 0.69 & 19.7 & 19.8 & 19.7 & & 0.1 & P. cognitus \\
\hline $3-2.5 \mathrm{M}$ & 0.69 & 19.2 & 19.2 & & & & P. gracilis \\
\hline $3-2.5 \mathrm{M}$ & 0.69 & 19.4 & 19.4 & 19.3 & & 0.1 & O. serratus \\
\hline $3-2.5 \mathrm{M}$ & 0.69 & 19.1 & 19.1 & 19.0 & & 0.0 & Mixed conodont \\
\hline $3-5 \mathrm{~L}$ & 0.25 & 19.1 & 19.1 & & & & Mixed conodont \\
\hline $3-3.0 \mathrm{M}$ & 0.22 & 18.8 & 19.4 & 18.3 & & 0.8 & Mixed conodont \\
\hline Grainstone & -0.70 & 19.1 & 19.1 & 19.0 & & 0.0 & D. suberectus \\
\hline Grainstone & -0.70 & 19.1 & 19.1 & 19.1 & & 0.0 & P. cognitus \\
\hline Grainstone & -0.70 & 19.0 & 19.1 & 19.0 & & 0.1 & O. serratus \\
\hline Carimona & -0.75 & 19.1 & 19.2 & 18.9 & & 0.2 & D. suberectus \\
\hline Carimona & -0.75 & 19.1 & 19.1 & 19.1 & & 0.0 & P. gracilis \\
\hline \multicolumn{8}{|l|}{ Rochester } \\
\hline $2-2 \mathrm{~L}$ & 8.5 & 19.3 & 19.2 & 19.3 & & 0.1 & Mixed conodont \\
\hline $2-0.5 \mathrm{~m}$ & 8.3 & 18.7 & 18.7 & & & & D. suberectus \\
\hline $2-0.5 \mathrm{~m}$ & 8.3 & 18.8 & 18.8 & 18.7 & & 0.1 & Mixed conodont \\
\hline $2-1.0 \mathrm{~m}$ & 8.2 & 18.3 & 18.3 & & & & D. suberectus \\
\hline $2-1.0 \mathrm{~m}$ & 8.2 & 18.6 & 18.6 & & & & P. undatus \\
\hline $2-1.0 \mathrm{~m}$ & 8.2 & 18.5 & 18.5 & & & & Mixed conodont \\
\hline $2-1.5 \mathrm{~m}$ & 7.4 & 18.2 & 18.2 & & & & D. suberectus \& O. serratus \\
\hline
\end{tabular}


Table 1. Continued.

\begin{tabular}{|c|c|c|c|c|c|c|c|}
\hline Sample ID & metres* & Avg. & $\delta^{18} \mathrm{O}$ & $\delta^{18} \mathrm{O}$ & $\delta^{18} \mathrm{O}$ & $\sigma$ & Description \\
\hline $2-4 \mathrm{~L}$ & 7.1 & 19.1 & 18.9 & \multirow[t]{4}{*}{19.4} & & \multirow[t]{4}{*}{0.4} & Mixed conodont \\
\hline $2-2.0 \mathrm{~m}$ & 7.0 & 19.0 & 19.0 & & & & P. aculeata \\
\hline $2-2.0 \mathrm{~m}$ & 7.0 & 18.1 & 18.1 & & & & P. undatus \\
\hline $2-2.0 \mathrm{~m}$ & 7.0 & 18.7 & 18.7 & & & & Mixed conodont \\
\hline $2-2.0 \mathrm{~m}$ & 7.0 & 18.8 & 19.0 & \multirow[t]{6}{*}{18.6} & & \multirow[t]{6}{*}{0.2} & D. suberectus \& O. serratus \\
\hline $2-2.5 \mathrm{~m}$ & 6.5 & 18.8 & 18.8 & & & & D. suberectus \\
\hline $2-2.5 \mathrm{~m}$ & 6.5 & 18.8 & 18.8 & & & & P. aculeata \\
\hline $2-2.5 \mathrm{~m}$ & 6.5 & 18.9 & 18.9 & & & & Mixed conodont \\
\hline $2-3.0 \mathrm{~m}$ & 6.0 & 18.7 & 18.7 & & & & D. suberectus \\
\hline $2-3.0 \mathrm{~m}$ & 6.0 & 18.0 & 18.0 & & & & Mixed conodont \\
\hline $2-8 \mathrm{~L}, 2-3.5 \mathrm{~m}$ & 5.4 & 19.2 & 19.3 & 19.1 & & 0.1 & Mixed conodont \\
\hline $2-9 \mathrm{~L}$ & 5.3 & 19.3 & 19.6 & 18.9 & & 0.5 & Mixed conodont \\
\hline $2-4 m$ & 5.0 & 19.3 & 19.2 & 19.4 & & 0.1 & Mixed conodont \\
\hline $2-5.0,2-5.5 \mathrm{~m}$ & 3.8 & 19.2 & 19.0 & 19.3 & & 0.2 & Mixed conodont \\
\hline $2-6.0 \mathrm{~m}$ & 2.6 & 19.4 & 19.3 & 19.5 & & 0.1 & Mixed conodont \\
\hline $2-6.5 m, 2-7.0 m$ & 1.7 & 19.2 & 19.4 & \multirow[t]{8}{*}{19.1} & & \multirow[t]{8}{*}{0.2} & D. suberectus \\
\hline $2-6.5 m, 2-7.0 m$ & 1.7 & 18.7 & 18.7 & & & & P. gracilis \\
\hline $2-6.5 m, 2-7.0 m$ & 1.7 & 19.0 & 19.0 & & & & Mixed conodont \\
\hline $2-7.5 \mathrm{~m}, 2-8.25 \mathrm{~m}$ & 1.0 & 19.1 & 19.1 & & & & D. suberectus \\
\hline $2-7.5 m, 2-8.25 m$ & 1.0 & 19.2 & 19.2 & & & & P. cognitus \\
\hline $2-7.5 \mathrm{~m}, 2-8.25 \mathrm{~m}$ & 1.0 & 18.6 & 18.6 & & & & P. gracilis \\
\hline $2-7.5 \mathrm{~m}, 2-8.25 \mathrm{~m}$ & 1.0 & 19.5 & 19.5 & & & & O. serratus \\
\hline $2-7.5 \mathrm{~m}, 2-8.25 \mathrm{~m}$ & 1.0 & 19.0 & 19.0 & & & & Mixed conodont \\
\hline 1.17 & 0.0 & 19.0 & 18.8 & \multirow{2}{*}{19.2} & & \multirow[t]{2}{*}{0.3} & D. suberectus \\
\hline 1.17 & 0.0 & 19.5 & 19.5 & & & & P. gracilis \\
\hline 1.17 & 0.0 & 19.0 & 19.0 & 18.9 & & 0.1 & Mixed conodont \\
\hline 1.16 & -0.1 & 19.5 & 19.5 & & & & P. cognitus \\
\hline 1.16 & -0.1 & 18.7 & 18.7 & & & & Mixed conodont \\
\hline 1.15 & -0.4 & 18.0 & 18.0 & & & & Mixed conodont \\
\hline 1.14 & -0.5 & 19.1 & 18.9 & 19.3 & & 0.3 & Mixed conodont \\
\hline 1.12 & -0.9 & 19.2 & 19.2 & & & & P. cognitus \\
\hline 1.12 & -0.9 & 18.9 & 18.9 & 18.9 & & 0.0 & Mixed conodont \\
\hline 1.11 & -1.1 & 19.7 & 19.7 & & & & Mixed conodont \\
\hline 10 & -1.3 & 18.8 & 18.8 & 18.8 & & 0.0 & D. suberectus \\
\hline 10 & -1.3 & 18.4 & 18.4 & & & & P. cognitus \\
\hline 10 & -1.3 & 18.8 & 18.7 & 18.9 & & 0.1 & P. gracilis \\
\hline 10 & -1.3 & 18.6 & 18.0 & 19.2 & & 0.8 & O. serratus \\
\hline 1.7 .3 & -1.6 & 18.9 & 18.8 & 19.0 & & 0.1 & B. compressa \\
\hline 1.7 .3 & -1.6 & 18.8 & 18.8 & 18.8 & & 0.1 & D. suberectus \\
\hline 1.7 .3 & -1.6 & 18.8 & 18.8 & 18.8 & & 0.0 & P. gracilis \\
\hline 1.7 .2 & -1.8 & 18.9 & 19.1 & 18.8 & & 0.2 & B. compressa \\
\hline 1.7 .2 & -1.8 & 18.5 & 18.6 & 18.5 & & 0.0 & D. suberectus \\
\hline 1.7 .2 & -1.8 & 18.8 & 18.8 & & & & P. gracilis \\
\hline 1.7 .2 & -1.8 & 18.5 & 18.6 & 18.5 & & 0.1 & P. ramosus \\
\hline 1.7 .1 & -1.8 & 19.3 & 19.3 & & & & B. compressa \\
\hline 1.7 .1 & -1.8 & 18.9 & 18.9 & 18.9 & & 0.0 & D. suberectus \\
\hline 1.7 .1 & -1.8 & 18.4 & 18.4 & & & & P. gracilis \\
\hline 1.7 .1 & -1.8 & 18.8 & 18.9 & 18.7 & & 0.1 & P. ramosus \\
\hline 1.5 & -2.0 & 19.1 & 19.1 & & & & B. compressa \\
\hline 1.5 & -2.0 & 19.7 & 19.7 & & & & D. suberectus \\
\hline 1.5 & -2.0 & 18.8 & 18.8 & & & & P. gracilis \\
\hline 1.5 & -2.0 & 18.3 & 18.0 & 18.6 & & 0.4 & P. ramosus \\
\hline 1.4 & -2.2 & 19.4 & 19.4 & & & & B. compressa \\
\hline 1.4 & -2.2 & 19.1 & 19.3 & 18.9 & & 0.3 & P. gracilis \\
\hline 1.4 & -2.2 & 18.7 & 18.6 & 18.8 & & 0.1 & P. ramosus \\
\hline 1.3 & -2.6 & 19.1 & 19.1 & & & & B. compressa \\
\hline 1.3 & -2.6 & 19.3 & 19.3 & 19.3 & & 0.0 & D. suberectus \\
\hline 1.3 & -2.6 & 18.9 & 18.9 & & & & P. gracilis \\
\hline 1.2 & -3.0 & 18.3 & 18.7 & 17.9 & & 0.5 & B. compressa \\
\hline 1.2 & -3.0 & 18.5 & 18.5 & & & & P. gracilis \\
\hline 1.1 & -3.4 & 18.8 & 18.8 & & & & Mixed conodont \\
\hline Dickeyville & & & & & & & \\
\hline DV 3-8.5 & 10.6 & 18.3 & 18.4 & 18.1 & & 0.2 & Mixed conodont \\
\hline DV 3-6.5 & 8.6 & 18.0 & 18.0 & & & & Mixed conodont \\
\hline DV $3.5 \mathrm{~m}$ & 5.6 & 18.3 & 18.3 & & & & P. undatus \\
\hline DV 0.0 & 2.1 & 19.3 & 19.3 & & & & Mixed conodont \\
\hline DV A18 & 1.8 & 18.7 & 18.7 & & & & Mixed conodont \\
\hline DV A15, A16, A17 & 1.6 & 19.5 & 19.5 & & & & Mixed conodont \\
\hline DV A 13 & 1.1 & 19.1 & 19.1 & & & & Mixed conodont \\
\hline DV A 13 & 1.1 & 19.0 & 19.0 & & & & D. suberectus \\
\hline DV A9 & 0.9 & 19.1 & 19.1 & & & & Mixed conodont \\
\hline DV A1 & 0.2 & 18.6 & 18.6 & & & & Mixed conodont \\
\hline DV P5 & -0.2 & 18.8 & 18.8 & & & & Mixed conodont \\
\hline DV P10 & -0.6 & 18.3 & 18.3 & & & & D. suberectus \\
\hline
\end{tabular}


Table 1. Continued.

\begin{tabular}{|c|c|c|c|c|c|c|c|}
\hline Sample ID & metres* & Avg. & $\delta^{18} \mathrm{O}$ & $\delta^{18} \mathrm{O}$ & $\delta^{18} \mathrm{O}$ & $\sigma$ & Description \\
\hline DV P10 & -0.6 & 19.5 & 19.5 & & & & Mixed conodont \\
\hline DV P11 & -0.7 & 19.0 & 19.0 & & & & Mixed conodont \\
\hline DV P 13 & -0.9 & 19.0 & 19.0 & & & & Mixed conodont \\
\hline \multicolumn{8}{|l|}{ Hagen } \\
\hline SAL-91HA-30 & 5.3 & 19.3 & 19.3 & & & & P. undatus \\
\hline SAL-91 HA-28 & 3.4 & 19.1 & 19.1 & & & & P. aculeata \\
\hline SAL-91 HA-28 & 3.4 & 19.1 & 19.1 & & & & Curtognathus sp. \\
\hline SAL-91 HA-27 & 2.1 & 18.6 & 18.6 & & & & P. aculeata \\
\hline SAL-91 HA-27 & 2.1 & 19.1 & 19.1 & & & & Curtognathus sp. \\
\hline SAL-91 HA-23 & -1.6 & 19.1 & 19.1 & & & & Curtognathus sp. \\
\hline SAL-91 HA-9 & -16.7 & 18.7 & 18.7 & & & & P. aculeata \\
\hline SAL-91 HA-9 & -16.7 & 19.5 & 19.5 & & & & Curtognathus sp. \\
\hline SAL-91 HA-7 & -18.0 & 18.7 & 18.7 & 18.8 & & 0.0 & P. aculeata \\
\hline SAL-91 HA-7 & -18.0 & 19.0 & 18.9 & 19.0 & & 0.1 & Curtognathus sp. \\
\hline SAL-91 HA-2 & -21.6 & 18.3 & 18.3 & & & & P. aculeata \\
\hline SAL-91 HA-2 & -21.6 & 19.4 & 19.4 & & & & Curtognathus sp. \\
\hline \multicolumn{8}{|l|}{ Red Mountain } \\
\hline RM 10.5 & 7.1 & 17.8 & 17.8 & & & & Mixed conodont \\
\hline RM 9.5 & 6.1 & 17.6 & 17.6 & & & & P. undatus \\
\hline RM 9.5 & 6.1 & 17.9 & 17.9 & & & & Mixed conodont \\
\hline RM 8.97 & 5.6 & 17.5 & 17.5 & & & & Mixed conodont \\
\hline RM 6 & 2.6 & 17.2 & 17.2 & & & & Mixed conodont \\
\hline RM 5.25 & 1.9 & 16.9 & 16.9 & & & & D. suberectus \\
\hline RM 5.25 & 1.9 & 18.0 & 18.0 & & & & P. undatus \\
\hline RM 5.25 & 1.9 & 17.7 & 17.7 & & & & Mixed conodont \\
\hline RM 5 & 1.6 & 17.7 & 17.7 & & & & Mixed conodont \\
\hline RM 3.5 & 0.1 & 17.7 & 17.7 & & & & Mixed conodont \\
\hline RM 3.45 & 0.1 & 17.9 & 17.9 & & & & P. undatus \\
\hline RM 3.45 & 0.1 & 16.6 & 16.8 & 16.5 & & 0.2 & Icriodella sp. \\
\hline RM 3.45 & 0.1 & $\mathbf{1 7 . 5}$ & 17.5 & & & & Mixed conodont \\
\hline RM 3 & -0.4 & 16.1 & 16.1 & & & & Mixed conodont \\
\hline RM 2 & -1.4 & 17.6 & 17.6 & & & & P. undatus \\
\hline RM 2 & -1.4 & 17.0 & 17.0 & & & & Mixed conodont \\
\hline RM 1.5 & -1.9 & 18.3 & 18.3 & & & & P. undatus \\
\hline RM 1.5 & -1.9 & 17.9 & 17.9 & & & & Mixed conodont \\
\hline RM 0.9 & -2.5 & 18.0 & 18.0 & & & & Mixed conodont \\
\hline RM 0 & -3.4 & 17.9 & 17.9 & & & & Mixed conodont \\
\hline \multicolumn{8}{|l|}{ Tidwell Hollow } \\
\hline $\mathrm{TH}+25$ & 25.1 & 18.1 & 18.3 & 17.9 & & 0.2 & Mixed conodont \\
\hline TH 25 & 25.0 & 19.1 & 19.5 & 18.7 & & 0.5 & Mixed conodont \\
\hline TH 22.85 & 22.9 & 18.4 & 18.4 & & & & Mixed conodont \\
\hline TH 20 & 20.0 & 18.4 & 18.2 & 18.6 & & 0.3 & Mixed conodont \\
\hline TH 18.5 & 18.5 & 18.1 & 18.2 & 18.0 & & 0.2 & Mixed conodont \\
\hline TH 13 & 13.0 & 18.1 & 18.1 & 18.0 & & 0.1 & Mixed conodont \\
\hline TH 11 & 11.0 & 17.8 & 18.1 & 17.6 & & 0.3 & Mixed conodont \\
\hline TH 8 & 8.0 & 17.3 & 17.3 & & & & Mixed conodont \\
\hline TH 7 & 7.0 & 19.7 & 19.7 & & & & Mixed conodont \\
\hline TH 4.5 & 2.5 & 19.1 & 19.1 & & & & Mixed conodont \\
\hline $\mathrm{TH}-9$ & -9.0 & 19.0 & 19.4 & 18.7 & & 0.5 & Mixed conodont \\
\hline $\mathrm{TH}-17$ & -17.0 & 18.7 & 18.7 & & & & Mixed conodont \\
\hline TH -19 & -19.0 & 18.5 & 18.6 & 18.4 & & 0.1 & Mixed conodont \\
\hline $\mathrm{TH}-21$ & -21.0 & 19.3 & 19.4 & 19.1 & & 0.2 & Mixed conodont \\
\hline $\mathrm{TH}-108.35$ & -108.4 & 19.1 & 19.1 & & & & Mixed conodont \\
\hline
\end{tabular}

* Detailed descriptions of outcrops from Alabama are available in Quinton et al. (2016), and for Hagen, VA, detailed descriptions are available in Young, Saltzmann \& Bergström (2005) and Leslie (1995).

${ }^{\dagger}$ metre above the Millbrig K-bentonite.

studied sections for which $\delta^{13} \mathrm{C}$ trends have not yet been documented. Carbon isotope measurements were performed on carbonate and trace organic matter for the Rochester and Decorah sections using methods described in Quinton et al. (2016a). All carbonate $\delta^{13} \mathrm{C}_{\text {carb }}$ measurements were performed on a ThermoFinnigan Delta Plus Dual Inlet isotope ratio mass spectrometer connected to a Kiel III Carbonate Interface. Analytical precision calculated from multiple analyses of NBS-19 run during the course of this study is $\pm 0.04 \%$ ( 1 standard deviation) for $\delta^{13} \mathrm{C}_{\text {carb }}$ and $\pm 0.06 \%$ for $\delta^{18} \mathrm{O}_{\text {carb }}$ (1 standard deviation). Organic carbon $\delta^{13} \mathrm{C}_{\text {org }}$ analyses were performed on decarbonated sample powders on a continuous flow ThermoFinnigan Delta Plus XL gas source mass spectrometer connected to an NA1500 EA chemical elemental analyser. Analytical precision is $\pm 0.1 \%$ ( 1 standard deviation) based on multiple analyses of an internal lab standard (Acetanilide). All $\delta^{13} \mathrm{C}$ values are reported in $\%$ on the Vienna PDB scale. 


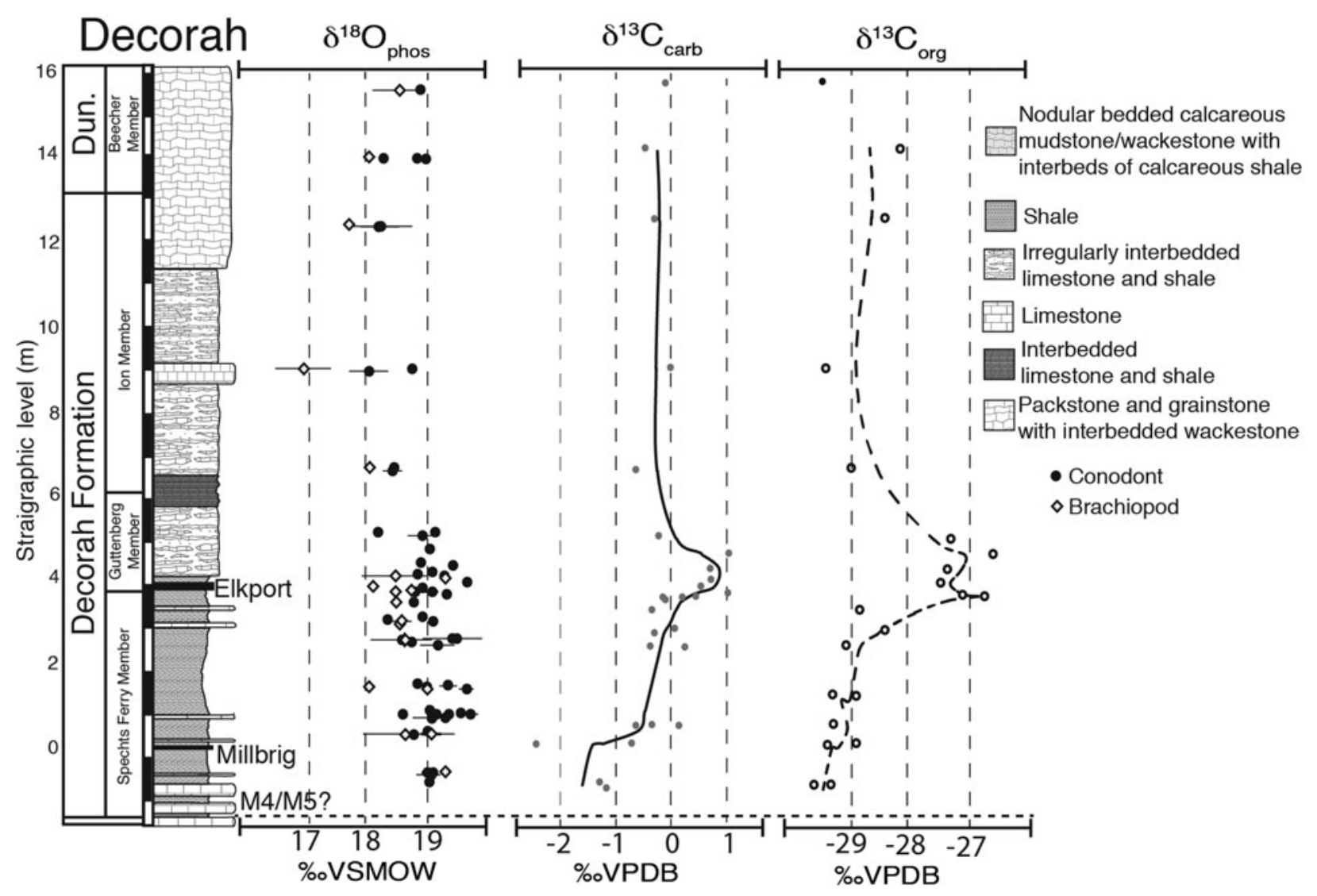

Figure 2. Average conodont and brachiopod $\delta^{18} \mathrm{O}$ results from Decorah, IO, plotted against bulk carbonate and organic carbon $\delta^{13} \mathrm{C}$ values. Carbon isotopic results are plotted with three-point moving averages. Averages are calculated from $\delta^{18} \mathrm{O}$ values of all replicate analyses of each silver phosphate crystal precipitation. Error bars on $\delta^{18} \mathrm{O}$ averages represent the standard deviation of replicate analyses for that sample. The $\sim 1 \%$ positive carbon isotope excursion above the Millbrig K-bentonite is interpreted as the GICE.

\section{Results}

Conodont apatite $\delta^{18} \mathrm{O}$ values for the latest Sandbian to early Katian range from $16.1 \%$ to $19.9 \%$, with an average of $18.8 \%$ (Table 1; Figs 2-4). This average corresponds to an estimated temperature of $31.3^{\circ} \mathrm{C}$ when using the temperature equation of Pucéat et al. (2010), a NBS-120c value of $21.7 \%$, assuming no change in $\delta^{18} \mathrm{O}_{\mathrm{sw}}$ (isotopic composition of seawater) due to ice volume effects, an ice-free $\delta^{18} \mathrm{O}_{\mathrm{sw}}$ value of $-1 \%$ VSMOW (Shackleton \& Kennett, 1975), and preservation of original isotopic values. Conodont $\delta^{18} \mathrm{O}$ regional averages for the Upper Mississippi Valley, Alabama and Virginia are $18.9 \%, 18.1 \%$ and $19 \%$ respectively (Fig. 4). In contrast, brachiopod apatite oxygen isotopic values average $\sim 17.4 \%$ and are generally lower (by $0.5-1.6 \%$ ) and more variable than values for cooccurring conodont elements (Figs 2-4; Supplementary Material Table 1 available at https://doi.org/10. $1017 / \mathrm{S} 0016756817000589)$. Average oxygen isotopic values from brachiopods would correspond to an estimated temperature of $37.3^{\circ} \mathrm{C}$ using the assumptions above.

Bulk carbonate $\delta^{13} \mathrm{C}_{\text {carb }}$ values from Rochester and Decorah range from $-3.9 \%$ to $1.0 \%$, and organic carbon $\delta^{13} \mathrm{C}_{\text {org }}$ values range from $-31.7 \%$ to $-25.4 \%$ (Figs 1 and 2). Bulk carbonate $\delta^{13} \mathrm{C}_{\text {carb }}$ values with an average of $-0.9 \%$ are relatively consistent through the sampled sections. At Decorah, both bulk carbonate and organic carbon results record a $\sim 1 \%$ positive excursion above the Millbrig K-bentonite.

\section{Discussion}

\section{4.a. Carbon isotopes}

North American carbon isotope stratigraphy is relatively well established for the Late Ordovician, with at least four globally recognized positive carbon isotope excursions. These excursions are used for chemostratigraphic correlation (e.g. Bergström et al. 2009, 2015), and the GICE, the second most prominent of these excursions, has also been proposed to represent the initiation of early Katian climatic cooling (Saltzman \& Young, 2005; Young et al. 2008). The stratigraphic position of the GICE has been previously identified in the Hagen (Young, Saltzmann \& Bergström, 2005), Tidwell Hollow and Red Mountain Expressway Quinton et al. (2016a), and Dickeyville (Wright et al. in press) sections. New bulk carbonate $\delta^{13} \mathrm{C}_{\text {carb }}$ results for the Decorah and Rochester sections allow for identification of the GICE and placement of $\delta^{18} \mathrm{O}$ results within the stable carbon isotope chemostratigraphic framework. At Decorah we interpret the $\sim 1 \%$ positive excursion starting at $3.7 \mathrm{~m}$ as the GICE. This interpretation would place the entirety of the excursion within 


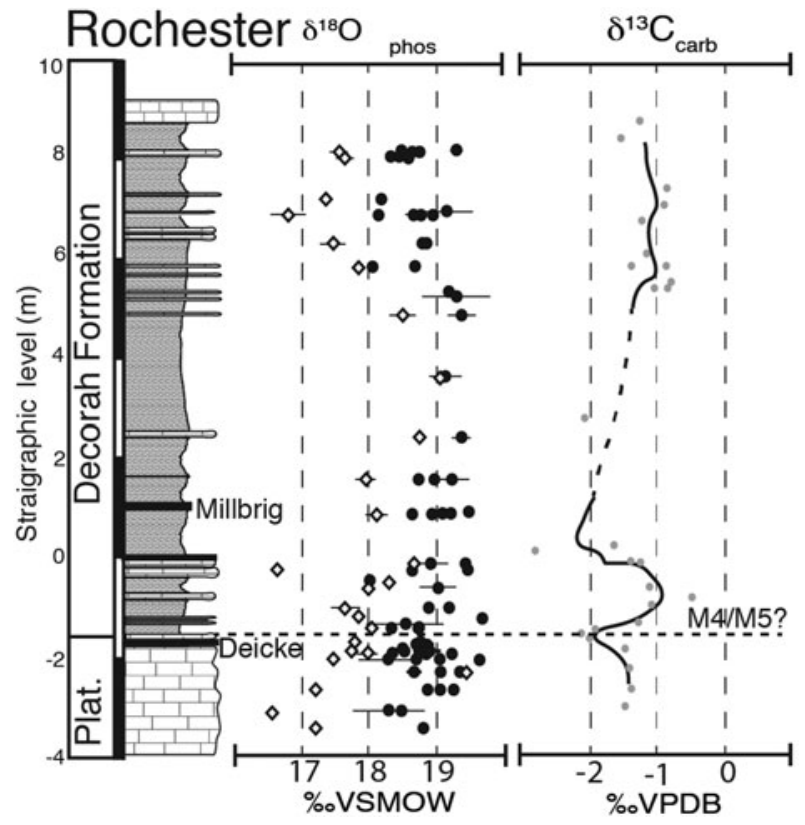

Figure 3. Conodont and brachiopod $\delta^{18} \mathrm{O}$ results from Rochester, MN, plotted against bulk carbonate $\delta^{13} \mathrm{C}_{\text {carb }}$ values. Carbon isotopic results are plotted with three-point moving averages. Averages are calculated from $\delta^{18} \mathrm{O}$ values of all replicate analyses of each silver phosphate crystal precipitation. Error bars on $\delta^{18} \mathrm{O}$ averages represent the standard deviation of replicate analyses for that sample. The low resolution of bulk carbonate $\delta^{13} \mathrm{C}_{\text {carb }}$ record prevents identification of the GICE with any confidence in this section. The Platteville Formation is abbreviated as Plat.

the Guttenberg Member. Results from Rochester record a $\sim 1 \%$ positive excursion below the Millbrig Kbentonite. However, given the stratigraphic position of this excursion and limited data above the Millbrig Kbentonite we cannot confidently identify the GICE at Rochester.

\section{4.b. Diagenetic considerations}

We interpret brachiopod $\delta^{18} \mathrm{O}$ results as having been shifted by diagenetic alteration. Linguliform brachiopods are benthic organisms and as such likely lived at a different position in the water column than most conodont taxa. Because temperatures generally decline with depth, this difference in habitat means brachiopods likely lived at temperatures lower than or similar to temperatures conodonts experienced. Thus, if primary oxygen isotopic values were preserved, brachiopods should have $\delta^{18} \mathrm{O}$ values the same as or higher than values in co-occurring conodont elements. In contrast to this prediction, brachiopod $\delta^{18} \mathrm{O}$ results are generally lower and more variable than values from conodonts in the same samples. These results are consistent with diagenetic overprints shifting brachiopod $\delta^{18} \mathrm{O}$ toward lower values. Note, though, that temperatures estimated from brachiopod $\delta^{18} \mathrm{O}$ values $\left(\sim 37.3^{\circ} \mathrm{C}\right)$ are significantly lower than burial temperatures estimated based on the CAI (lower than 50$80^{\circ} \mathrm{C}$ ) of co-occurring conodont elements (Epstein,

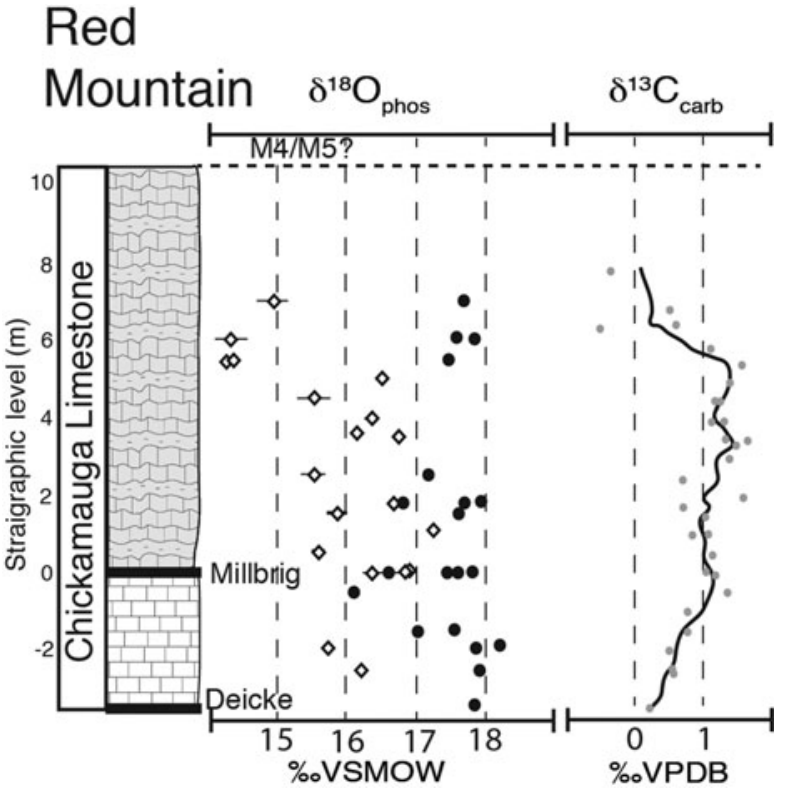

Figure 4. Conodont and brachiopod $\delta^{18} \mathrm{O}$ results from Red Mountain Expressway, AL, plotted against bulk carbonate $\delta^{13} \mathrm{C}_{\text {carb }}$ values from Quinton et al. (2016a) . Averages are calculated from $\delta^{18} \mathrm{O}$ values of all replicate analyses of each silver phosphate crystal precipitation. Error bars on $\delta^{18} \mathrm{O}$ averages represent the standard deviation of replicate analyses for that sample.

Epstein \& Harris, 1977). Thus, brachiopod oxygen isotopic values were not completely reset during burial.

The relatively modest overprint on brachiopod oxygen isotopic values and the relative resistance to alteration of conodont apatite suggests that conodont $\delta^{18} \mathrm{O}$ results likely primarily reflect palaeoenvironmental conditions. Supporting this argument, isotopic values and trends across most of the sampled sections argue against diagenesis as an explanation for observed patterns as it is unlikely that $\delta^{18} \mathrm{O}$ values would be consistently altered across multiple sections separated by $>1000 \mathrm{~km}$ and influenced by different secondary processes and with different burial histories. Further, conodont $\delta^{18} \mathrm{O}$ values yield a temperature estimate of 30 $32{ }^{\circ} \mathrm{C}$, that falls near the range of modern tropical sea surface temperatures and is comparable to predictions from numerical climate models for Katian equatorial sea surface temperatures (e.g. Herrmann et al. 2004; Pohl et al. 2014). Results from Red Mountain Expressway are the one exception to these general observations and are discussed below.

\section{4.c. Local influences on $\delta^{18} \mathrm{O}$ values}

Conodont oxygen isotopic results from this study combined with previous results (Buggisch et al. 2010; Rosenau, Herrmann \& Leslie, 2012) provide the first record of $\delta^{18} \mathrm{O}$ values across the SE region of Laurentia's epicontinental sea during the Late Ordovician. With these results, it is possible to test for systematic variations in $\delta^{18} \mathrm{O}_{\mathrm{sw}}$ values and patterns in the region using the Deicke and Millbrig K-bentonites as 


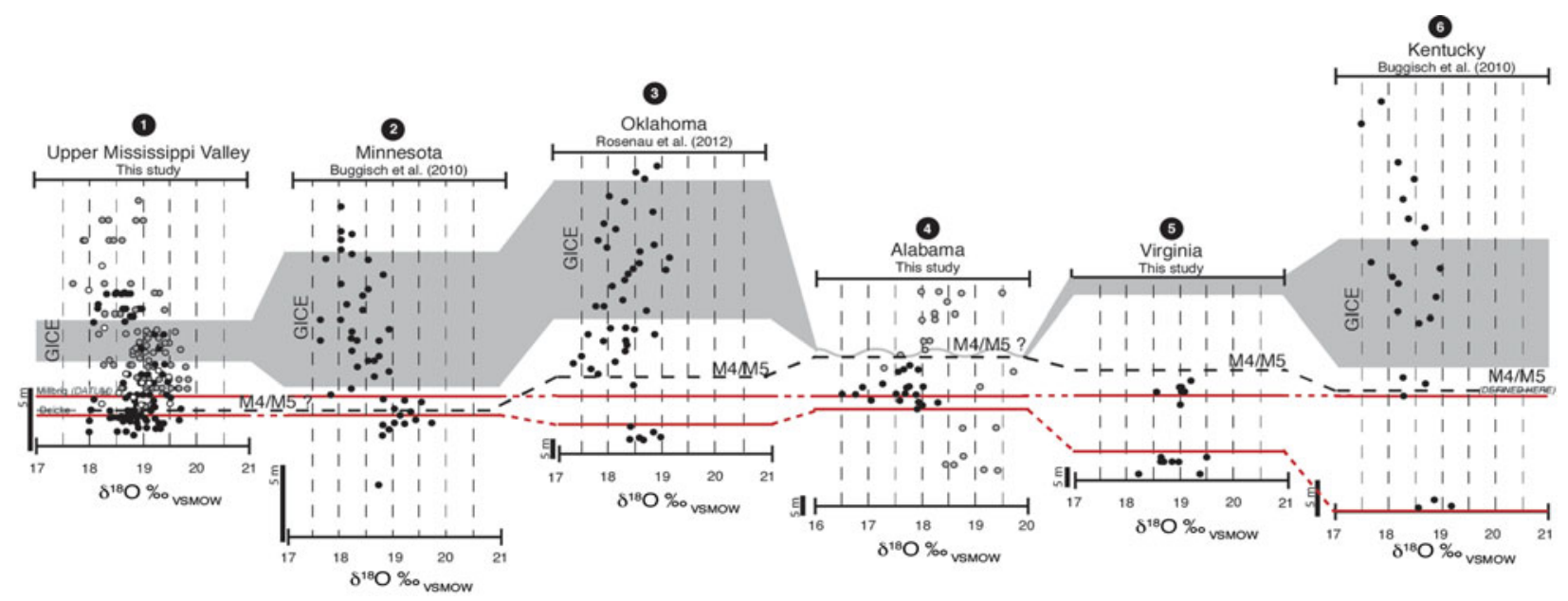

Figure 5. (Colour online) Compilation of Late Ordovician species-specific and mixed conodont $\delta^{18} \mathrm{O}$ values from the Upper Mississippi Valley (this study), Minnesota (from Buggisch et al. 2010), Oklahoma (Rosenau, Herrmann \& Leslie, 2012), Alabama (this study), Virginia (this study) and Kentucky (Buggisch et al. 2010), with Deicke and Millbrig K-bentonites (red lines) as planes of correlation. The Upper Mississippi Valley data from this study includes results from Decorah (black circles), Rochester (grey circles) and Dickeyville (open circles). Oxygen isotopic values from Alabama include results from Tidwell Hollow (grey circles) and Red Mountain Expressway (black circles). Regional averages are 18.9\%o for the Upper Mississippi Valley, 18.7\%o for Minnesota, $18.3 \%$ o for Oklahoma, 18.1\%o for Alabama, $19 \%$ for Virginia and 18.4\%o for Kentucky. The M4/M5, the GICE, and K-bentonites are identified in Minnesota (Buggisch et al. 2010), Alabama Quinton et al. (2016a), Virginia (Young, Saltzmann \& Bergström, 2005) and Kentucky (Buggisch et al. 2010). It should be noted that while the GICE has been labelled in Oklahoma (Rosenau, Herrmann \& Leslie, 2012) others have questioned this identification (Westrop et al. 2015). The GICE is identified in the Upper Mississippi Valley based on $\delta^{13} \mathrm{C}$ results from this study (see online Supplementary Material available at https://doi.org/10.1017/S0016756817000589). The M4/M5 boundary (identified in Kentucky) is correlated with the contact between the Platteville and Decorah formations in the Upper Mississippi Valley (following Holland \& Patzkowsky, 1996; Kolata, Huff \& Bergström, 2001); this correlation of the M4/M5 shows that the sequence boundary is regionally diachronous assuming that none of the K-bentonites has been misidentified or miscorrelated. All $\delta^{18} \mathrm{O}$ values are normalized to an NBS-120c value of $21.7 \%$.

stratigraphic markers for purposes of regional correlation (Fig. 5). First, it should be noted that there is significant scatter in the $\delta^{18} \mathrm{O}$ data. Scatter appears to be a common feature of high-resolution conodont oxygen isotopic records and likely reflects some combination of intra- and inter-annual temperature/salinity differences, and taxonomic differences. These differences are made more noticeable by analytical uncertainty that is several times larger in phosphate measurements than carbonate measurements (Quinton \& MacLeod, 2014; Quinton et al. 2016b). The documented variability is of a similar magnitude within all the study sections and is observed in both species-specific and mixed conodont assemblage oxygen isotopic results. Further, generated $\delta^{18} \mathrm{O}$ records for each section include multiple measurements for many sample horizons, maximizing potential to capture the $\delta^{18} \mathrm{O}$ average for that time interval. For these reasons, observed variability should not bias our results, but it does highlight the importance of testing for patterns in average $\delta^{18} \mathrm{O}$ values over many samples rather than interpreting differences between adjacent samples.

The strata exposed in the Upper Mississippi Valley and Kentucky were deposited on the shallow Galena and Lexington carbonate platforms respectively. Exposures in Alabama and Virginia were deposited along the margin of the Taconic Foreland Basin while those from Oklahoma were deposited in the Arkoma basin. Despite differences in position and depth in the epicontinental sea, regional $\delta^{18} \mathrm{O}$ averages are relatively consistent (Upper Mississippi Valley: 18.9\%, 1б: 0.5; Kentucky: $18.4 \%$, 1б: 0.4; Tidwell Hollow Alabama: $18.7 \%$, 1б: 0.7; Virginia: $19 \%, 1 \sigma: 0.4$; and Oklahoma: $18.3 \%, 1 \sigma: 0.4$ ) and record a similar pattern of decreasing values in the early Katian.

Similarly, $\delta^{18} \mathrm{O}$ values do not significantly vary across proposed temperature- and salinity-defined aquafacies (Holmden et al. 1998) as would be expected if local differences in freshwater input and evaporation/precipitation rates exerted a significant influence on $\delta^{18} \mathrm{O}_{\mathrm{sw}}$ values. Based on differences in $\mathrm{Nd}$ and $\mathrm{C}$ isotopic values among regions, Holmden et al. (1998) suggested that Laurentia's epicontinental sea contained three distinct aquafacies: the Midcontinent, Taconic and Southern (Fig. 1). These authors suggested that basin morphology and geographic patterns in the source and amount of freshwater runoff controlled the presence of each aquafacies. Both environmental factors can have a significant influence on the oxygen isotopic composition of regional and local seawater. As such, measured $\delta^{18} \mathrm{O}$ values from these aquafacies are expected to be distinct if local environmental influences on $\delta^{18} \mathrm{O}_{\mathrm{sw}}$ were significant. In contrast to this prediction, measured $\delta^{18} \mathrm{O}$ values from the Midcontinent aquafacies (e.g. Upper Mississippi Valley), Southern aquafacies (e.g. Kentucky) and Taconic aquafacies (e.g. Virginia) are generally consistent around 18.4$19 \%$, arguing that $\delta^{18} \mathrm{O}_{\mathrm{sw}}$ values did not vary systematically due to local environmental conditions that impart signals observed in $\mathrm{Nd}$ and $\mathrm{C}$ trends in the region. 
Therefore, it seems values and trends in $\delta^{18} \mathrm{O}$ values primarily reflect regional patterns.

Oxygen isotopic results from Red Mountain Expressway, at an average of $17.5 \%$, are the one exception to basin-wide similarity among conodont $\delta^{18} \mathrm{O}$ values. During the Late Ordovician, Red Mountain Expressway was situated at the highest palaeolatitude $\left(\sim 30^{\circ} \mathrm{S}\right)$ and represents one of the greatest water depths of the studied sections, yet $\delta^{18} \mathrm{O}$ values are up to $1 \%$ lower at this site than others. The lower values could be interpreted as an indication of local water $4-5^{\circ} \mathrm{C}$ warmer than elsewhere, but this interpretation is difficult to reconcile with the Red Mountain Expressway's palaeogeographic and palaeoceanographic position. Diagenetic alteration provides an alternative explanation for the low values at Red Mountain Expressway. Exposures in Alabama were subjected to the highest burial temperatures, in excess of $110^{\circ} \mathrm{C}$ as indicated from the conodont elements with a CAI of 3, of all the sections sampled. Brachiopod $\delta^{18} \mathrm{O}$ values are also generally lowest and the degree of offset between conodont $\delta^{18} \mathrm{O}$ values and brachiopod values at Red Mountain Expressway is the greatest among the sections studied. Both observations are consistent with a more pervasive diagenetic overprint at Red Mountain Expressway than other sections.

\section{4.d. Oxygen isotopes and 'cool-water' carbonates}

Our compilation of $\delta^{18} \mathrm{O}$ records argues against 'coolwater' models that invoke climatic cooling to explain the history of Sandbian-Katian carbonate deposition on Laurentia. Conodont $\delta^{18} \mathrm{O}$ values do not increase across the interval; in fact, if anything they decrease on average by $1.5 \%$ following the M4/M5. As noted above, there are no basin-scale gradients suggestive of strong local influences on $\delta^{18} \mathrm{O}_{\text {sw }}$ values that compromise temperature inferences, and diagenetic overprints do not seem to be a major concern except at the Red Mountain Expressway section. Further, additional considerations about global fluctuations in $\delta^{18} \mathrm{O}_{\mathrm{sw}}$ due to possible increases in ice volume are not required. Glacial growth, which can cause an increase in $\delta^{18} \mathrm{O}_{\mathrm{sw}}$ values, and climatic cooling can both contribute to recorded $\delta^{18} \mathrm{O}$ values, but both shift values in the same direction (i.e. increased $\delta^{18} \mathrm{O}$ values). Distinguishing between the relative contributions of either on observed $\delta^{18} \mathrm{O}$ trends only becomes necessary when attempting to estimate the amount of temperature and/or ice volume change. We suggest that the lack of oxygen isotopic increase in conodonts through the sections studied indicates that water temperature was not a dominant factor controlling the stratigraphic trends among early Katian carbonates in SE Laurentia.

The tectonic model is the only one that does not predict an increase in $\delta^{18} \mathrm{O}$ values and thus is favoured by these data. In a tectonic scenario, the change in carbonate lithology resulted from increased turbidity, terrigenous input, and/or changing circulation patterns in response to basin deepening across SE Laurentia and uplift of the Taconic Mountains (e.g. Ettensohn, 2010). Reduced light penetration due to increased water turbidity could explain the shift to skeletally supported fabrics and the absence of chlorozoan fauna (both characteristics of early Katian 'cool-water' carbonates). Detailed analyses of the carbonates themselves would be required to distinguish among the relative importance of different proximate forcing mechanisms (terrigenous input, nutrient increase, changing circulation) brought about by the Taconic uplift.

\section{4.e. Possible palaeoenvironmental implications of early Katian $\delta^{18} \mathrm{O}$ decrease}

Our results show no evidence of cooling in the Sandbian - early Katian, but they are consistent with diachronous warming across SE Laurentia during this interval. All regions record a $\sim 1-0.5 \%$ decrease in average $\delta^{18} \mathrm{O}$ values following the M4/M5, but the timing of this increase varies among sections. This observation becomes apparent when the compiled oxygen isotope data are separated into a pre-M4/M5, a post-M4/M5 and a GICE + post-GICE time-slice using the stratigraphic position of these boundaries and the Deicke K-bentonite to scale the data (Fig. 6).

Stratigraphically below the M4/M5 sequence boundary, oxygen isotopic values from the Upper Mississippi Valley, Oklahoma, Alabama, Virginia and Kentucky overlap and record similar scatter around an average of $\sim 19 \%$. Values from the Red Mountain Expressway are markedly lower but believed to be diagenetically altered as discussed above. Following the M4/M5, oxygen isotopic values become more variable and values from marginal settings (Oklahoma and Alabama) decrease and diverge from the interior settings (Upper Mississippi Valley). During the GICE, values from all sections again overlap and record similar fluctuations but at a lower average $(\sim 0.5 \%)$ than prior to the M4/M5. The divergence in $\delta^{18} \mathrm{O}$ values between marginal and interior sections following the M4/M5 could reflect diachronous warming across the region, with warming first along the margin of the epicontinental sea, then in interior settings.

On the other hand, it is possible to explain the diachronous decrease in oxygen isotopic values through a change in the moisture balance (and therefore $\delta^{18} \mathrm{O}_{\mathrm{sw}}$ ) within the basin or an influx of open ocean waters (with different $\delta^{18} \mathrm{O}_{\mathrm{sw}}$ than the water it replaces) into a semirestricted shallow epicontinental sea characterized by net evaporation. Net evaporation within the epicontinental sea has been proposed (Railsback et al. 1990; Ludvigson \& Jacobson, 1996) and would result in a ${ }^{18} \mathrm{O}$ enrichment in basin interior settings. In such a scenario, marginal settings would be expected to experience decreasing oxygen isotopic values first as they are closest to the open ocean, an interpretation that fits well with the observed sequence of $\delta^{18} \mathrm{O}$ changes.

While it is not possible to distinguish between these alternatives without additional data (e.g. $\mathrm{Nd}$ to 

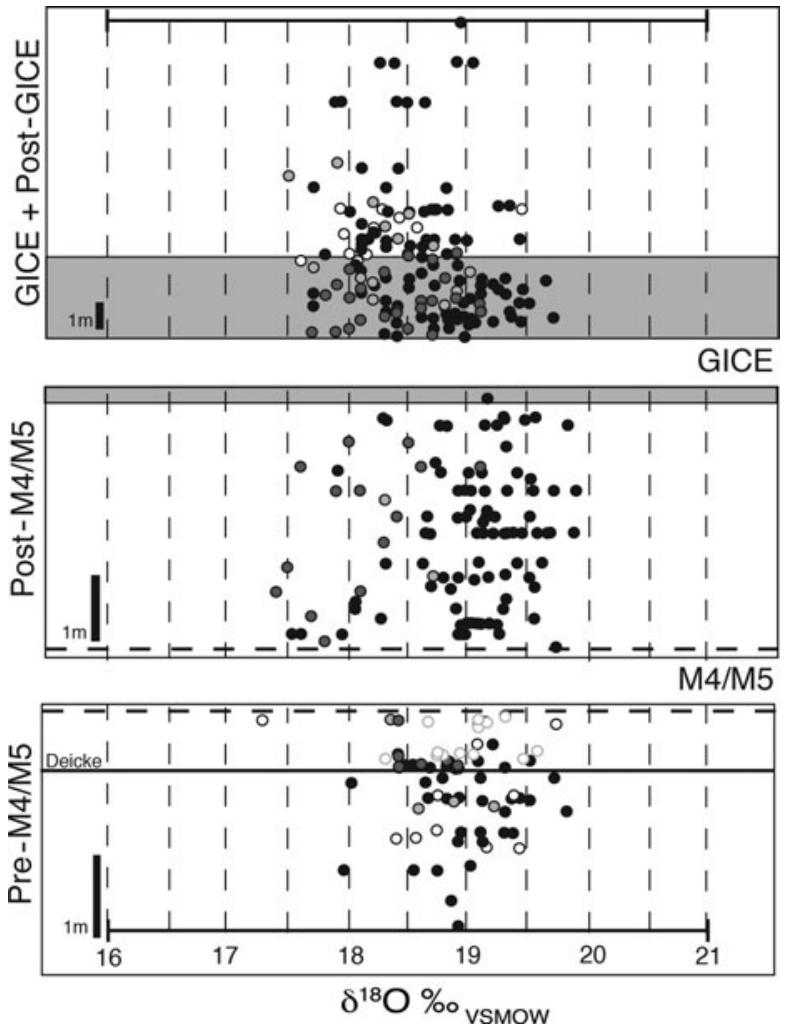

Figure 6. Comparison of new and previously documented conodont oxygen isotopic values from the Upper Mississippi Valley (black circles), Oklahoma (dark grey circles), Alabama (hollow circles), Virginia (open grey circles) and Kentucky (light grey circles) at three different time-slices in the Late Ordovician. Data from the Red Mountain Expressway section are not plotted due to suspected diagenetic overprint. All sections are scaled and plotted against stratigraphic thickness in the Rochester section. Metre scale for each time-slice refers to stratigraphic thickness in the Rochester section. For the pre-M4/M5 interval, all data are scaled to the position of the M4/M5 and the Deicke K-bentonite. For the post-M4/M5 interval, all data are scaled based on the stratigraphic position of the M4/M5 and the base of the GICE. For the GICE and post-GICE interval, all data were scaled based on the thickness of the GICE in each section.

constrain changes in circulation patterns), we favour an interpretation invoking warming. As argued above, there is no compelling evidence to indicate significant $\delta^{18} \mathrm{O}_{\mathrm{sw}}$ gradients. Additionally, independent geochemical evidence ( $\mathrm{Nd}$ and $\mathrm{C}$ ) suggests that marginal settings like Alabama were well mixed with open-ocean water (Holmden et al. 1998). Therefore, values from Tidwell Hollow should be lower than those from interior settings where evaporative enrichment of ${ }^{18} \mathrm{O}$ is expected. Contrary to this prediction, $\delta^{18} \mathrm{O}$ values from the Tidwell Hollow section are generally higher than those observed from the interior section (i.e. Kentucky). Finally, average temperature estimates assuming an open ocean $\delta^{18} \mathrm{O}_{\mathrm{sw}}$ value (i.e. not evaporatively enriched) at $\sim 32^{\circ} \mathrm{C}$ match predicted Katian sea surface temperatures for the region (e.g. Herrmann et al. 2004; Pohl et al. 2014).

\section{Conclusions}

New conodont oxygen isotope data from this study provide the first record of $\delta^{18} \mathrm{O}$ variability across $\mathrm{SE}$ North America from which to test for local environmental influences and, thereby, constrain regional $\delta^{18} \mathrm{O}$ trends. Oxygen isotopic values are consistent (i.e. exhibit a similar range and degree of scatter) across bathymetric features as well as across proposed water mass boundaries for the sampled interval, suggesting that local environmental factors (e.g. evaporation, precipitation and/or freshwater influx) did not result in significant $\delta^{18} \mathrm{O}_{\mathrm{sw}}$ differences across the region. We argue that the diachronous $\sim 1.5 \%$ decrease in oxygen isotopic values across the M4/M5 likely reflects regional warming.

Based on these arguments, new oxygen isotopic records do not support the 'cool-water' interpretation for Sandbian - early Katian carbonates. Our results show no evidence of cooling across the M4/M5 boundary and so we argue that cooling was not the dominant factor controlling the fabrics and fossil content of early Katian carbonates in SE Laurentia. Instead, the shift in carbonate lithology across the M4/M5 seems better explained by a tectonically driven increase in turbidity in the basin. It should be noted that discriminating among the alternative hypotheses is possible because the compiled $\delta^{18} \mathrm{O}$ data provide temporal and spatial control on a continental scale, a result that highlights the importance of documenting patterns with relatively high resolution from multiple sections when inferring palaeoenvironmental forcing.

Acknowledgements. We thank Katie Meierdiercks and Ian Knoerl at James Madison University and Claire Jones, Zach Wright, Bryan Mogrovejo, John Michael Callen and Cody Schulte at Louisiana State University for assistance with fieldwork and laboratory work. Additionally, we thank Cheryl Kelley and Shannon Haynes for assistance with analyses at the University of Missouri Stable Isotope and Biogeochemistry Laboratory, and two anonymous reviewers. This research was funded by NSF EAR 1323444 to K.G.M., NSF EAR 1324577 to S.A.L., and NSF EAR 1324954 to A.D.H.

\section{Supplementary material}

To view supplementary material for this article, please visit https://doi.org/10.1017/S0016756817000589

\section{References}

Bassett, D., MacLeod, K. G., Miller, J. F. \& Ethington, R. L. 2007. Oxygen isotopic composition of biogenic phosphate and temperature of Early Ordovician seawater. Palaios 22, 98-103.

Bergström, S. M., Chen, X., Gutiérrez-Marco, J. C. \& Dronov, A. 2009. The new chronostratigraphic classification of the Ordovician System and its relations to major regional series and stages and to $\delta^{13} \mathrm{C}$ chemostratigraphy. Lethaia 42, 97-107.

Bergström, S. M., Saltzman, M. R., Leslie, S. A., Ferretti, A. and Young, S. A. 2015. Trans-Atlantic 
application of the Baltic Middle and Upper Ordovician carbon isotope zonation. Estonian Journal of Earth Sciences 64, 8-12.

Bergström, S. M., Schmitz, B., Saltzman, M. R. \& Huff, W. D. 2010. The Upper Ordovician Guttenberg $\delta^{13} \mathrm{C}$ excursion (GICE) in North America and Baltoscandia: occurrence, chronostratigraphic significance, and paleoenvironmental relationships. In The Ordovician System (eds S. C. Finney \& W. B. N. Berry), pp. 37-67. Geological Society of America, Special Paper no. 466.

Brett, C., Mclaughlin, P. I., Cornell, S. R. \& Baird, G. C. 2004. Comparative sequence stratigraphy of two classic Upper Ordovician successions, Trenton Shelf (New York-Ontario) and Lexington Platform (Kentucky-Ohio): implications for eustasy and local tectonism in eastern Laurentia. Palaeogeography, $\mathrm{Pa}$ laeoclimatology, Palaeoecology 210, 295-329.

Buggisch, W., JOACHIMSKI, M. M., LeHNERT, O., Bergström, S. M., Repetski, J. E. \& Webers, G. F. 2010. Did intense volcanism trigger the first Late Ordovician icehouse? Geology 38, 327-30.

Dronov, A. 2013. Late Ordovician cooling event: evidence from the Siberian craton. Palaeogeography, Palaeoclimatology, Palaeoecology 389, 87-95.

Elrick, M., Reardon, D., Labor, W., Martin, J., Desrochers, A. \& Pope, M. 2013. Orbital-scale climate change and glacioeustasy during the early Late Ordovician (pre-Hirnantian) determined from $\delta^{18} \mathrm{O}$ values in marine apatite. Geology 41, 775-8.

Emerson, N. R., Ludvigson, G. A., Witzke, C. L., Schneider, C. L., González, L. A. \& CARpenter, S. J. 2005. STOP 5 - The Fred Carlson CO., LLC Locust Quarry west of Locust Iowa. In Facets of the Ordovician Geology of the Upper Mississippi Valley Region (eds G. A. Ludvigson \& B. J. Bunker), pp. 95-109. Iowa Geological Survey Guidebook Series no. 24.

Epstein, A. G., Epstein, J. B. \& Harris, L. D. 1977. Conodont color alteration - an index to organic metamorphism. US Geological Survey Professional Paper no. $995,-27$ pp.

EtTensohn, F. R. 2010. Origin of Late Ordovician (midMohawkian) temperature: water conditions on southeastern Laurentia: glacial or tectonic? In The Ordovician System (eds S. C. Finney \& W. B. N. Berry), pp. 163-75. Geological Society of America Special Paper no. 466 .

Ettensohn, F. R., Hohman, J. C., Kulp, M. A. \& Rast, N. 2002. Evidence and implications of possible farfield responses to Taconian orogeny: Middle-Late Ordovician Lexington Platform and Sebree Trough, eastcentral United States. Southeastern Geology 41, 1-36.

Hall, J. C., Bergström, S. M. \& Schmidt, M. A. 1986. Conodont biostratigraphy of the Middle Ordovician Chickamauga Group and related strata of the Alabama Appalachians. In Depositional History of the Middle Ordovician of the Alabama Appalachians: For the 23rd Annual Field Trip, Alabama Geological Society (eds J. Benson \& C. W. Stock), pp. 61-80.

Haynes, J. T. 1994. The Ordovician Deicke and Millbrig Kbentonite beds of the Cincinnati Arch and the Southern Valley and Ridge Province. Geological Society of America, Special Paper no. 290, 80 pp.

Herrmann, A. D., Haupt, B. J., Patzkowsky, M. E., Seidov, D. \& Slingerland, R. L. 2004. Response of Late Ordovician paleoceanography to changes in sea level, continental drift, and atmospheric $p \mathrm{CO}_{2}$ : potential causes for long-term cooling and glaciations. $\mathrm{Pa}$ - laeogeography, Palaeoclimatology, Palaeoecology 210, 385-401.

Herrmann, A. D., MacLeod, K. G. \& Leslie, S. A. 2010. Did a volcanic mega-eruption cause global cooling during the Late Ordovician? Palaios 25, 831-6.

Holland, S. M. \& Patzkowsky, M. E. 1996. Sequence stratigraphy and long-term paleoceanographic changes in the Middle and Upper Ordovician of the eastern United States. In Paleozoic Sequence Stratigraphy: Views from the North American Craton (eds B. J. Wittke, G. A. Ludvigson \& J. F. Day), pp. 117-28. Geological Society of America, Special Paper no. 306.

Holmden, C., Creaser, R. A., Muehlenbachs, K., Leslie, S. A. \& BergströM, S. M. 1998. Isotopic evidence for geochemical decoupling between ancient epeiric seas and bordering oceans: implications for secular curves. Geology 26, 567-70.

Hughes, K. P., MacLeod, K. G., Haynes, S. J., Quinton, P. C., Martin, E. E. \& Ethington, R. 2015. A paired neodymium and oxygen isotopic perspective on paleoceanographic changes across the Dubuque/Maquoketa contact in the Late Ordovician Laurentian Seaway. Stratigraphy 12, 275-85.

Jeprsson, L. \& ANEhUs, R. 1995. A buffered formic acid technique for conodont extraction. Journal of Paleonto$\log y 69,790-4$.

Jeppsson, L., Anehus, R. \& Fredholm, D. 1999. The optimal acetate buffered acetic acid technique for extracting phosphatic fossils. Journal of Paleontology 73, 946-72.

Joachimski, M. M., Breisig, S., Buggisch, W., Talent, J. A., Mawson, R., Gereke, M., Morrow, J. R., Day, J. \& Weddige, K. 2009. Devonian climate and reef evolution: insights from oxygen isotopes in apatite. Earth and Planetary Science Letters 284, 599-609.

Kolata, D. R., Huff, W. D. \& Bergström, S. M. 1996. Ordovician K-bentonites of eastern North America. Geological Society of America, Special Paper no. 313, - 84 pp.

Kolata, D. R., Huff, W. D. \& Bergström, S. M. 1998. Nature and regional significance of unconformities associated with the Middle Ordovician Hagan Kbentonite complex in the North America midcontinent. Geological Society of America Bulletin 110, 723-39.

Kolata, D. R., Huff, W. D. \& Bergström, S. M., 2001. The Ordovician Sebree Trough: an oceanic passage to the Midcontinent United States. Geological Society of America Bulletin 113, 1067-8.

Kolodny, Y. \& LuZ, B., 1991. Oxygen isotopes in phosphates of fossil fish - Devonian to recent. In TAYLOR, H. T. J. (ed.), Stable Isotope Geochemistry: A Tribute to Samuel Epstein, pp. 65-76. San Antonio, TX, Geochemical Society.

Kolodny, Y., Luz, B. \& Navon, O. 1983. Oxygen isotope variations in phosphate of biogenic apatites: I. Fish bone apatite: rechecking the rules of the game. Earth and Planetary Science Letters 64, 398-404.

Laporte, D. F., Holmden, C., Patterson, W. P., Prokopiuk, T. \& Eglington, B. M. 2009. Oxygen isotope analysis of phosphate: improved precision using TC/EA CF-IRMS. Journal of Mass Spectrometry 44, 879-90.

LAVOIE, D. 1995. A Late Ordovician high-energy temperate-water carbonate ramp, southern Quebec, Canada: implications for Late Ordovician oceanography, Sedimentology, 42, 95-116.

Lavoie, D. \& Asselin, E. 1998. Upper Ordovician facies in the Lac Saint-Jean outlier, Québec (eastern Canada): 
palaeoenvironmental significance for Late Ordovician oceanography, Sedimentology 45, 817-32.

Leslie, S. A. 1995. Upper Middle Ordovician conodont biofacies distribution patterns in eastern North America and northwestern Europe: evaluations using the Deicke, Millbrig and Kinnekulle K-bentonite beds as time planes. Ph.D. thesis, The Ohio State University, Columbus, OH, USA. Published thesis.

Leslie, S. A. 2000. Mohawkian (Upper Ordovician) conodonts of Eastern North America and Baltoscandia. Journal of Paleontology 74, 1122-47.

LesLie, S. A. \& Bergström, S. M. 2003. Widespread, prolonged late Middle to Late Ordovician upwelling in North America: a proxy record of glaciation? Comment. Geology 31, e28-e29.

LONGINELLI, A. 1965. Oxygen isotopic composition of orthophosphate from shells of living and fossil marine organisms. Nature 211, 923-7.

Ludvigson, G. \& JACOBSON, S. 1996. Carbonate component chemostratigraphy and depositional history of the Ordovician Decorah Formation, Upper Mississippi Valley. In Paleozoic Sequence Stratigraphy: Views from the North American Craton (eds B. J. Wittke, G. A. Ludvigson \& J. F. Day), pp. 67-86. Geological Society of America, Special Paper 306.

Luz, B., Kolodny, Y. \& Kovach, J. 1984. Oxygen isotope variations in phosphate of biogenic apatites, III. Conodonts. Earth and Planetary Science Letters 69, 25562.

MacLeOd, K. G. 2012. Conodonts and the paleoclimatological and paleoecological applications of phosphate $\delta^{18} \mathrm{O}$ measurements. In Reconstructing Earth's DeepTime Climate - The State of the Art in 2012 (eds L. C. Ivany \& B. T. Huber), pp. 69-84. Paleontological Society Papers 18.

Pedley, M. \& Carannante, G., 2006. Cool-water carbonate ramps: a review. In Cool-water Carbonates: Depositional Systems and Palaeoenvironmental Controls (eds M. Pedley \& C. Carannante), pp. 1-9. Geological Society of London, Special Publication 255.1.

Pohl, A., Donnadieu, Y., Hir, G. L., Buoncristiani, J. F. \& Vennin, E. 2014. Effect of the Ordovician paleogeography on the (in)stability of the climate. Climate Past 10, 2053-66.

Pope, M. C. \& ReAd, J. F., 1997. High-resolution stratigraphy of the Lexington limestone (late Middle Ordovician), Kentucky, U.S.A.: a cool-water carbonate-clastic ramp in a tectonically active foreland basin. In Coolwater-carbonates (eds P. Noel \& J. A. D. Clarke), pp. 410-29. SEPM Special Publication 56.

Pope, M. C. \& Steffen, J. B. 2003. Widespread, prolonged late Middle to Late Ordovician upwelling in North America: a proxy record of glaciation? Geology 31, 636.

Pucéat, E., Joachimski, M. M., Bouilloux, A., Monna, F., Bonin, A, Motreuil, S., Morinière, P., HÉnard, S., Mourin, J., Dera, G. \& Quesne, D., 2010. Revised phosphate-water fractionation equation reassessing paleotemperatures derived from biogenic apatite. Earth and Planetary Science Letters 298, $135-42$.

Quinton, P. C., Herrmann, A. D., Leslie, S. A. \& MACLeOD, K. G. 2016a. Carbon cycling across the southern margin of Laurentia during the Late Ordovician. Palaeogeography, Palaeoclimatology, Palaeoeco$\operatorname{logy}$ 458. doi:10.1016/j.palaeo.2015.08.020.

Quinton, P. C., Leslie, S. A., Herrmann, A. D. \& MacLeOD, K. G. 2016b. Effects of extraction protocols on the oxygen isotope composition of conodont elements. Chemical Geology 431, 36-43.

Quinton, P. C. \& MacLeOD, K. G. 2014. Oxygen isotopes from conodont apatite of the midcontinent US: implications for Late Ordovician climate evolution. Palaeogeography, Palaeoclimatology, Palaeoecology 404, 57-66.

Railsback, L. B., Ackerly, S. C., Anderson, T. F. \& Cisne, J. L. 1990. Palaeontological and isotope evidence for warm saline deep waters in Ordovician oceans. Nature 343, 156-9.

Rosenau, N. A., Herrmann, A. D. \& Leslie, S. A. 2012. Conodont apatite $\delta^{18} \mathrm{O}$ values from a platform margin setting, Oklahoma, USA: implications for initiation of Late Ordovician icehouse conditions. Palaeogeography, Palaeoclimatology, Palaeoecology 315-316, 172-80.

Saltzman, M. R. \& Young, S. A. 2005. Long-lived glaciation in the Late Ordovician? Isotopic and sequencestratigraphic evidence from western Laurentia. Geology 33, 109-12.

SAMANKASSOU, E. 2002. Cool-water carbonates in a paleoequatorial shallow-water environment: the paradox of the Aurenig cyclic sediments (Upper Pennsylvanian, Carnic Alps, Austria-Italy) and its implications. Geo$\log y$ 30, 655-8.

Sell, B. K., Samson, S. D., Mitchell, C. E., McLaughlin, P. J., Koenig, A. E. \& LeSLiE, S. A. 2015. Stratigraphic correlations using trace elements in apatite from Late Ordovician (Sandbian-Katian) K-bentonites of eastern North America. Geological Society of American Bulletin 127, 125974.

Shackleton, N. J. and Kennett, J. P. 1975. Late Cenozoic oxygen and carbon isotopic changes at DSDP Site 284; implications for glacial history of the Northern Hemisphere and Antarctica. Initial Reports of the Deep Sea Drilling Project 29, 801-7.

Sun, Y., Joachimski, M. M., Wignall, P. B., Yan, C., Chen, Y., JiAng, H., Wang, L. \& LaI, X., 2012. Lethally hot temperatures during the early Triassic greenhouse, Science 338, 366-70.

Torsvik, T. H. \& Cocks, R. M. 2013. New global palaeogeographical reconstructions for the Early Palaeozoic and their generation. In Early Palaeozoic Biogeography and Palaeogeography (ed. D.A.T. Harper \& T. Servais), pp. 5-24. Geological Society of London, Memoirs 38.

Trotter, J. A., FitzGerald, J. D., KokKonen, H. \& BARNES, C. R., 2007. New insights into the ultrastructure, permeability, and integrity of conodont apatite determined by transmission electron microscopy. Lethaia, 40: $97-110$.

Trotter, J. A., Williams, I. S., Barnes, C. R., Lecuyer, C. \& NicolL, R. S. 2008. Did cooling oceans trigger Ordovician biodiversification? Evidence from conodont thermometry. Science 321, 550-4.

Wenzel, B., LeCuYer, C. \& JoACHimski, M. M. 2000. Comparing oxygen isotope records of Silurian calcite and phosphate- $\delta^{18} \mathrm{O}$ compositions of brachiopods and conodonts. Geochimica et Cosmochimica Acta 64, 185972.

Westrop, S. R., Amati, L., Brett, C. E., Swisher, R. E., Carlucci, J. R., Goldman, D., Leslie, S. A. \& BurkHAlter, R. 2015. The more the merrier? Reconciling sequence stratigraphy, chemostratigraphy, and multiple biostratigraphic indices in the correlation of the Katian reference section, central Oklahoma. Stratigraphy, abstracts 12, 139.

Wright, Z., Quinton, P. C., Martin, E., Leslie, S. A., Macleod, K. G. \& Herrmann, A. D. In press. 
Neodymium isotope ratios and a positive $\delta^{13} \mathrm{C}$ excursion: interpreting the connection between oceanographic and climate changes during the early Late Ordovician of Laurentia. Stratigraphy.

Young, S. A., Saltzman, M. R. \& Bergström, S. M. 2005. Upper Ordovician (Mohawkian) carbon isotope $\left(\delta^{13} \mathrm{C}\right)$ stratigraphy in eastern and central North America: regional expression of a perturbation of the global carbon cycle. Palaeogeography, Palaeoclimatology, Palaeoecology 222, 53-76.

Young, S. A., Saltzman, M. R., Bergström, S. M., Leslie, S. A. \& Xu, C., 2008. Paired $\delta^{13} C_{\text {carb }}$ and $\delta^{13} \mathrm{C}_{\text {org }}$ records of Upper Ordovician (Sandbian-Katian) carbonates in North America and China: implications for paleoceanographic change. Palaeogeography, $\mathrm{Pa}$ laeoclimatology, Palaeoecology 270: 166-78. 